\title{
Vaginal Cancer by AJCC v8 Stage
}

National Cancer Institute

\section{Source}

National Cancer Institute. Vaginal Cancer by A/CC v8 Stage. NCI Thesaurus. Code C139657.

A term that refers to the staging of vaginal cancer according to the American Joint Committee on Cancer, 8th edition. This staging system applies to all carcinomas of the vagina. There is no AJCC staging system for mucosal melanoma of the vagina. (from AJCC 8th Ed.) 